\title{
PREVALÊNCIA E CARACTERIZAÇÃO DE FERIDAS CRÔNICAS EM IDOSOS ASSISTIDOS NA ATENÇÃO BÁSICA
}

\section{PREVALENCE AND CHARACTERIZATION OF CHRONIC WOUNDS IN ELDERLY PERSONS ASSISTED IN PRIMARY CARE}

\section{PREVALENCIA Y CARACTERIZACIÓN DE FERIDAS CRÓNICAS EN ANCIANOS ASISTIDOS EN LA ATENCIÓN BÁSICA}

\author{
Chrystiany Plácido de Brito Vieira ${ }^{1}$ \\ Alessandra Silveira Furtado ${ }^{2}$ \\ Priscilla Carvalho Dias de Almeida ${ }^{2}$ \\ Maria Helena Barros Araújo Luz ${ }^{3}$ \\ Antônio Francisco Machado Pereira
}

Como citar este artigo: Vieira CPB, Furtado AS, Almeida PCD, Luz MHBA, Pereira AFM. Prevalência e caracterização de feridas crônicas em idosos assistidos na atenção básica. Rev baiana enferm. 2017;31(3):e17397.

\begin{abstract}
Objetivo: analisar a prevalência e a caracterização de feridas crônicas em idosos assistidos na atenção básica e descrever as características sociodemográficas e clínicas desses idosos. Método: pesquisa transversal com 339 idosos, realizada entre janeiro e maio de 2016. Resultados: 55,8\% tinham de 60 a 70 anos, 67,3\% eram mulheres, sem escolaridade (44\%), casadas (51,9\%) e com uma ou mais doenças (91,7\%). A prevalência foi de 8\% (IC 95\% 5,0-10,9), sendo 5\% lesões por pressão e 2,9\% úlceras vasculogênicas. Verificou-se associação da ocorrência de ferida crônica com escolaridade $(p=0,03)$ e escore do Mini-Exame do Estado Mental $(p=0,000)$ e diferença estatística na média da idade entre os idosos com lesões e os que não apresentavam lesão $(p=0,000)$. Conclusão: houve baixa prevalência de feridas crônicas na população estudada. A região sacral foi a mais atingida e $48,2 \%$ das lesões apresentavam tecido de granulação e tinham média de tempo de existência de três anos.
\end{abstract}

Descritores: Idoso. Ferimentos e lesões. Atenção primária.

Objective: to analyze the prevalence and characterization of chronic wounds in the elderly assisted in primary care and to describe the sociodemographic and clinical characteristics of these elderly patients. Method: cross-sectional study with 339 elderly people, conducted between January and May 2016. Results: 55.8\% were from 60 to 70 years old, 67.3\% were women, with no schooling (44\%), married (51.9\%) and with one or more diseases (91.7\%). The prevalence was 8\% (95\% CI, 5.0-10.9), with 5\% pressure lesions and 2.9\% vasculogenic ulcers. There was an association between the occurrence of chronic wounds with schooling $(p=0.03)$ and Mini-Mental State Examination (MMSE) score $(p=0.000)$ and statistical difference in mean age between the elderly with lesions and those with no lesion $(P=0.000)$. Conclusion: there was a low prevalence of chronic wounds in the studied population. The sacral

\footnotetext{
Enfermeira. Doutora em Enfermagem. Docente do Departamento de Enfermagem da Universidade Federal do Piauí. Teresina, Piauí, Brasil. chrystianyplacido@yahoo.com Enfermeiras. Teresina, Piauí, Brasil.

Enfermeira. Doutora em Enfermagem. Docente do Departamento de Enfermagem da Universidade Federal do Piauí. Teresina, Piauí, Brasil.

Enfermeiro. Docente do Departamento de Enfermagem da Universidade Federal do Piaúi. Teresina, Piaú, Brasil.
} 
region was the most affected and $48.2 \%$ of the lesions presented granulation tissue and had an average time of existence of three years.

Descriptors: Elderly. Wounds and injuries. Primary care.

Objetivo: analizar prevalencia y caracterización de beridas crónicas en ancianos asistidos en la atención básica y describir sus características sociodemográficas y clinicas. Método: investigación trasversal, realizada entre enero y mayo de 2016 con 339 ancianos. Resultados: El 55,8\% tenía entre 60 y 70 años, mujeres (67,3\%), sin escolaridad (44\%), casadas (51,9\%), con una o más enfermedades (91,7\%). La prevalencia fue del 8\% (IC 95\% 5,0-10,9), siendo $5 \%$ lesiones por presión y 2,9\% úlceras vasculogénicas. Se verificó asociación de ocurrencia de herida crónica con escolaridad $(p=0,03)$, puntuación del Mini-Examen del Estado Mental $(p=0,000)$ y diferencia estadística en la media de edad entre los ancianos con lesiones y los que no presentaban lesión ( $p=0,000)$. Conclusión: hubo baja prevalencia de heridas crónicas en la población estudiada. La región sacral fue la más afectada y el 48,2\% de las lesiones presentaban tejido de granulación y un promedio de tiempo de existencia de tres años.

Descriptores: Ancianos. Heridas y lesiones. Atención primaria.

\section{Introdução}

O crescimento da população idosa é um fenômeno natural, irreversível e de caráter mundial. Ocorre em ritmos e velocidades diferentes nas várias regiões do mundo, sendo que os aspectos que interferem na expansão da população com essa faixa etária são as mudanças nas condições de vida e os avanços da medicina no combate a enfermidades, os quais reduzem as mortalidades precoces, como também promovem a longevidade. Neste sentido, com o envelhecimento da população, consequentemente há aumento global de doenças crônicas, sendo previsível também elevação concomitante no número de pessoas com lesões secundárias à patologia de base ${ }^{(1)}$.

A maioria das feridas crônicas está associada às condições da população idosa, incluindo doença vascular, insuficiência venosa, hipertensão e diabetes mellitus. Ainda permanecem questões fundamentais sobre o efeito do envelhecimento na cicatrização e nos mecanismos de reparo das lesões, bem como a regeneração dos tecidos em idosos ${ }^{(2)}$. Dentre os tipos de feridas crônicas mais frequentemente encontrados nos serviços da atenção básica de saúde, encontram-se as úlceras vasculogênicas (UV) e as lesões por pressão (LP), que geralmente são de longa evolução e de resposta terapêutica variável.

As UV são agravos clínicos relevantes, afetando cerca de 600.000 americanos anualmente, sendo mais prevalentes na população idosa. Alcança taxa superior a $4 \%$ em pessoas com idade acima de 65 anos. Além disso, a incidência global da UV está prevista para aumentar de forma significativa, em conjunto com o aumento do número da população idosa, pois está diretamente associada às patologias crônicas que surgem com o envelhecimento ${ }^{(3)}$.

A prevalência de LP em pessoas com 60 anos de idade ou mais é maior que em pessoas com menor idade, isso devido à presença dos principais fatores de risco para o desenvolvimento dessa lesão, dentre eles os considerados intrínsecos, como a própria idade e a presença de algumas doenças crônicas prevalentes nessa parcela da população ${ }^{(4-5)}$. Em pesquisa realizada em Teresina, Piauí, Brasil, foram avaliados 30 pacientes acamados sob assistência domiciliária da Estratégia Saúde da Família (ESF), com idades entre 18 e 108 anos, com 80\% de idosos, apontando prevalência de UP de $13,33 \%{ }^{(5)}$.

Desse modo, faz-se necessário conhecimento da problemática na população assistida para planejamento das ações de cuidado. Diante disso, o objetivo deste trabalho foi analisar a prevalência e a caracterização de feridas crônicas em idosos assistidos na atenção básica e descrever as características sociodemográficas e clínicas desses idosos. 


\section{Método}

Trata-se de pesquisa quantitativa e transversal, realizada na atenção básica da cidade de Teresina (PI), Brasil, com idosos assistidos pelas equipes da ESF das três Diretorias Regionais de Saúde (DRS) do município, no período de janeiro a maio de 2016.

A amostra calculada foi de 339, com a fórmula $\mathrm{n}=\mathrm{z}^{2}$.p.(1- p) / $\mathrm{e}^{2}$, onde: $z$ é 1,96, $p$ prevalência de feridas crônicas na população idosa de $17 \%{ }^{(6)}$; e margem de erro de 4\%. Para seleção da amostra, aplicou-se o processo de amostragem estratificada proporcional em três etapas. Na primeira etapa, realizou-se a distribuição proporcional pelo quantitativo de idosos de cada DRS, ficando 92 idosos participantes da DRS Centro/ Norte, 109 da Sul e 138 da Leste/Sudeste. Na segunda etapa, foi realizada a distribuição proporcional do número de Unidades Básicas de Saúde (UBS) a ser sorteado por DRS: 6 na Centro/Norte, 9 na Sul e 10 na Leste/Sudeste. Na terceira e última etapa, distribuiu-se proporcionalmente o quantitativo de idosos dentro das UBS, aleatoriamente selecionadas por meio do software $\mathrm{R}$ (Project for Statistical Computing) versão 3.0.2 para compor a amostra.

Os idosos foram sorteados aleatoriamente e tinham que ter moradia fixa na área adstrita à ESF das UBS sorteadas. Como critérios de exclusão, estabeleceu-se os idosos não encontrados no domicílio após três tentativas de agendamento da entrevista, fosse por motivo de viagem ou por não residir mais no endereço.

A coleta de dados foi feita pelas pesquisadoras, por meio de entrevistas no domicílio. Utilizou-se como instrumentos o Mini-Exame do Estado Mental (MEEM), o Instrumento de Dados Sociodemográficos, Econômicos e Clínicos, a Escala de Katz e a Escala de Push. Assim, a coleta de dados foi constituída de três etapas:

Etapa 1 - Avaliação cognitiva: o MEEM foi aplicado para avaliação da condição cognitiva e de responder à entrevista. Neste estudo, foi utilizada a versão adaptada no $\mathrm{Brasil}^{(7)}$, cujos pontos de corte adotados foram de 13 para analfabetos, 18 para baixa e média escolaridade e 26 para alta escolaridade. Caso o idoso apresentasse escore abaixo desses valores, a entrevista era realizada com o cuidador ou familiar responsável.

Etapa 2 - Dados sociodemográficos, econômicos e clínicos: o instrumento foi elaborado para levantar informações sociodemográficas, econômicas e clínicas relacionadas à presença e aos tipos de patologias crônicas, uso de medicamentos, hábitos de vida e independência funcional para realização das Atividades Básicas de Vida Diária (ABVD), classificadas segundo a Escala de Katz, versão adaptada ${ }^{(8)}$, em índices de 0 a 6.

Etapa 3 - Avaliação da ferida: caso o idoso apresentasse ferida do tipo LP ou UV com mais de três meses de evolução, era aplicada a última parte do instrumento acima para avaliação da lesão. Em caso de LP, considerou-se o total de até 4 (quatro) lesões por participante, pelo fato de o idoso poder apresentar múltiplas lesões em diferentes regiões corporais. Nesta situação, avaliava-se as lesões mais antigas, sendo utilizada para identificação, a nomenclatura ferida 1 (F1), ferida 2 (F2) e assim por diante. Na classificação do estágio, as LP foram classificadas segundo o consenso internacional da National Pressure Ulcer Advisory Pane ${ }^{(9)}$ como categorias I, II, III e IV, lesões não classificáveis e lesão tissular profunda. As UV foram classificadas, de acordo com o critério de perda tissular ${ }^{(10)}$, em perda parcial de tecido ou espessura parcial e em perda total de tecido ou espessura total. Na caracterização da ferida, quanto à cicatrização, foi aplicado o Pressure Ulcer Scale for Healing (PUSH), adaptado e validado para a língua portuguesa ${ }^{(11)}$.

Inicialmente, foi realizada a organização dos dados mediante a revisão manual dos questionários. Os dados foram digitados no programa Microsoft Excel 2013 e depois importados para o programa Statistical Package for the Social Sciences (SPSS) for Windows (versão 19.0). Posteriormente, foram realizadas análises descritivas, distribuição, frequência e estatística inferencial.

Para todas as análises, fixou-se o nível de significância de 0,05. Para verificar associação 
e tornar viável a aplicação dos testes, algumas variáveis foram recodificadas. Realizou-se o teste qui-quadrado de Pearson e exato de Fischer. A força das associações foi calculada por meio da Odds Ratio (OR) com intervalo de confiança de 95\%. Para comparação de médias, primeiramente, foi aplicado o teste de aderência à normalidade de Kolmogorov-Smirnov e, em seguida, o teste T de Student para a variável idade. A discussão foi feita à luz dos conhecimentos produzidos e publicados sobre o tema.

Quanto aos aspectos éticos, o estudo atende aos fundamentos contidos na Resolução n. 466/2012, com obtenção de parecer favorável n. 1.144.315 do Comitê de Ética em Pesquisa da Universidade Federal do Piauí.

\section{Resultados}

Participaram do estudo 339 idosos distribuídos nas três DRS: leste/sudeste $(40,7 \%)$, sul $(32,2 \%)$ e norte $(27,1 \%)$. Na Tabela 1 , verifica-se que a média de idade foi 71,1 anos $( \pm 8,9), 55,8 \%$ na faixa etária de 60 a 70 anos, com predominância do sexo feminino (67,3\%), sem escolaridade (44\%), com companheiro $(51,9 \%)$ e morando com cônjuge, filhos e/ou netos (71,7\%). Verifica-se ainda que $85 \%$ recebiam entre um a três salários mínimos, sendo $72,3 \%$ aposentados e 20\% desenvolviam alguma atividade remunerada.

Tabela 1 - Caracterização sociodemográfica e econômica dos idosos entrevistados. Teresina, Piauí, Brasil - 2016. ( $\mathrm{n}=339)$

\begin{tabular}{|c|c|c|c|c|c|c|}
\hline Variáveis & $\mathbf{n}$ & $\%$ & Média & $\begin{array}{l}\text { Desvio } \\
\text { Padrão }\end{array}$ & $\begin{array}{c}\text { Intervalo de } \\
\text { Confiança } 95 \%\end{array}$ & $\begin{array}{l}\text { Mínima } \\
\text { e Máxima }\end{array}$ \\
\hline Idade (em anos) & & & 71,1 & 8,9 & $70,1-72,0$ & $60-106$ \\
\hline $60-70$ & 189 & 55,8 & & & & \\
\hline $71-80$ & 95 & 28,0 & & & & \\
\hline 81 e mais & 55 & 16,2 & & & & \\
\hline \multicolumn{7}{|l|}{ Sexo } \\
\hline Masculino & 111 & 32,7 & & & & \\
\hline Feminino & 228 & 67,3 & & & & \\
\hline Escolaridade & & & 2,64 & 3,0 & $2,3-2,9$ & $0-15$ \\
\hline Sem & 149 & 44,0 & & & & \\
\hline Baixa (1-3 anos) & 61 & 18,0 & & & & \\
\hline Média (4-7 anos) & 91 & 26,8 & & & & \\
\hline Alta (8 anos e mais) & 38 & 11,2 & & & & \\
\hline \multicolumn{7}{|l|}{ Estado civil } \\
\hline Solteiro/separado & 72 & 21,2 & & & & \\
\hline Casado/união estável & 176 & 51,9 & & & & \\
\hline Viúvo & 91 & 26,9 & & & & \\
\hline \multicolumn{7}{|l|}{ Com quem mora } \\
\hline Sozinho(a) & 25 & 7,4 & & & & \\
\hline $\begin{array}{l}\text { Com cônjuge/companheiro, } \\
\text { filho(s) e/ou netos }\end{array}$ & 243 & 71,7 & & & & \\
\hline Com parentes/amigos & 71 & 20,9 & & & & \\
\hline \multicolumn{7}{|l|}{ Renda familiar $(*)$} \\
\hline$<1$ & 5 & 1,5 & & & & \\
\hline $1-3$ & 288 & 85,0 & & & & \\
\hline$>3$ & 46 & 13,5 & & & & \\
\hline \multicolumn{7}{|l|}{ Aposentadoria } \\
\hline Sim & 245 & 72,3 & & & & \\
\hline Não & 94 & 27,7 & & & & \\
\hline
\end{tabular}


Tabela 1 - Caracterização sociodemográfica e econômica dos idosos entrevistados. Teresina, Piauí, Brasil - 2016. ( $n=339)$

(conclusão)

\begin{tabular}{l|c|c|c|c|c|c}
\hline Variáveis & $\mathbf{n}$ & $\mathbf{\%}$ & Média & $\begin{array}{c}\text { Desvio } \\
\text { Padrão }\end{array}$ & $\begin{array}{c}\text { Intervalo de } \\
\text { Confiança 95\% }\end{array}$ & $\begin{array}{c}\text { Mínima } \\
\text { e Máxima }\end{array}$ \\
\hline $\begin{array}{l}\text { Desenvolve alguma atividade } \\
\text { Nenhuma }\end{array}$ & 89 & 26,3 & & \\
Atividade doméstica & 179 & 52,8 & & \\
Trabalho remunerado & 68 & 20,0 & \\
Trabalho voluntário & 3 & 0,9 &
\end{tabular}

Fonte: Elaboração própria.

(*) SM = Salário mínimo $(\mathrm{R} \$ 880,00)$.

De acordo com a Tabela 2, a maioria $(91,7 \%)$ possuía uma ou mais doenças, sendo as mais prevalentes: hipertensão arterial $(70,1 \%)$, hipercolesterolemia (31,5\%), diabetes mellitus (29,9\%), doença cardiovascular $(13,8 \%)$ e doença vascular periférica (10,3\%). Quanto à medicação, 87,3\% faziam uso de uma ou mais, em média 2,8 medicações $( \pm 1,6)$, dentre as quais as mais citadas foram os anti-hipertensivos (73,2\%), os hipoglicemiantes $(30,5 \%)$, os cardiovasculares $(16,6 \%)$ e psicotrópicos (10,5\%). Na avaliação do MEEM, constatou-se que a maioria dos idosos apresentou média do escore acima dos pontos de corte para a ausência, baixa e média escolaridades, exceto alta escolaridade, que apresentou média de $24,7 \%( \pm 5,1)$. Verifica-se ainda que a grande maioria $(83,5 \%)$ era independente para todas as funções, 81,7\% tinha locomoção preservada, $98,8 \%$ alimentavam-se via oral e 59\% sem restrições alimentares, com média de 4,4 refeições ao dia $( \pm 0,9)$ e $36,9 \%$ com relato de perda de peso. A maioria relatou não fumar e não beber, respectivamente, 43,4\% e 67,6\%. No entanto, apenas 23,9\% faziam atividade física (Tabela 2).

Tabela 2 - Caracterização dos idosos entrevistados de acordo com variáveis clínicas e hábitos de vida.

Teresina, Piauí, Brasil - 2016. (n=339)

(continua)

\begin{tabular}{|c|c|c|c|c|c|c|}
\hline Variáveis & $\mathbf{n}$ & $\%$ & Média & $\begin{array}{l}\text { Desvio } \\
\text { Padrão }\end{array}$ & $\begin{array}{c}\text { Intervalo de } \\
\text { Confiança } \\
95 \%\end{array}$ & $\begin{array}{l}\text { Mínimo } \\
\text { e Máxim }\end{array}$ \\
\hline \multicolumn{7}{|l|}{ Presença de doenças } \\
\hline Uma ou mais & 311 & 91,7 & & & & \\
\hline Nenhuma doença & 28 & 8,3 & & & & \\
\hline Faz uso de medicação & & & 2,8 & 1,6 & $2,6-3,0$ & $1-9$ \\
\hline Sim & 296 & 87,3 & & & & \\
\hline Não & 43 & 12,7 & & & & \\
\hline \multicolumn{7}{|c|}{ Mini-Exame do Estado Mental } \\
\hline Sem escolaridade & & & 15,8 & 6,6 & $14,7-16,9$ & $0-26$ \\
\hline Baixa escolaridade & & & 21,3 & 3,8 & $20,3-22,2$ & $3-27$ \\
\hline Média escolaridade & & & 22,2 & 6,0 & $20,9-23,4$ & $0-30$ \\
\hline Alta escolaridade & & & 24,7 & 5,1 & $23,0-26,4$ & $4-30$ \\
\hline
\end{tabular}

KATZ

Independente em todas as seis

funções

Independentes em cinco funções $\quad 13 \quad 3,8$

e dependente em uma função

Independente em quatro funções

e dependente em duas funções

$6 \quad 1,8$

Independente em três funções

$6 \quad 1,8$ 
Tabela 2 - Caracterização dos idosos entrevistados de acordo com variáveis clínicas e hábitos de vida. Teresina, Piauí, Brasil - 2016. (n=339) (conclusão)

\begin{tabular}{|c|c|c|c|c|c|c|}
\hline Variáveis & $\mathbf{n}$ & $\%$ & Média & $\begin{array}{l}\text { Desvio } \\
\text { Padrão }\end{array}$ & $\begin{array}{c}\text { Intervalo de } \\
\text { Confiança } \\
95 \%\end{array}$ & $\begin{array}{l}\text { Mínimo } \\
\text { e Máximo }\end{array}$ \\
\hline $\begin{array}{l}\text { Independente em duas funções } \\
\text { e dependente em quatro funções }\end{array}$ & 6 & 1,8 & & & & \\
\hline $\begin{array}{l}\text { Independente em uma função } \\
\text { e dependente em cinco funções }\end{array}$ & 9 & 2,6 & & & & \\
\hline Dependente em todas as funções & 16 & 4,7 & & & & \\
\hline \multicolumn{7}{|l|}{ Atividade/ locomoção } \\
\hline Preservada/ deambula & 277 & 81,7 & & & & \\
\hline Deambula com ajuda & 38 & 11,2 & & & & \\
\hline Não deambula/ cadeirante & 5 & 1,5 & & & & \\
\hline Não deambula/ acamado & 19 & 5,6 & & & & \\
\hline \multicolumn{7}{|l|}{ Alimentação } \\
\hline Oral & 335 & 98,8 & & & & \\
\hline $\begin{array}{l}\text { Sonda Nasoenteral/Sonda } \\
\text { nasogástrica/Gastrostomia }\end{array}$ & 4 & 1,2 & & & & \\
\hline \multicolumn{7}{|l|}{ Ingestão alimentar } \\
\hline Sem restrição & 200 & 59,0 & & & & \\
\hline Restrição alimentar & 126 & 37,2 & & & & \\
\hline Dificuldade de ingestão & 13 & 3,8 & & & & \\
\hline Número de refeições (dia) & & & 4,4 & 0,9 & $4,3-4,5$ & $1-6$ \\
\hline \multicolumn{7}{|l|}{ Relato Variação de peso } \\
\hline Não & 121 & 35,7 & & & & \\
\hline Ganho & 93 & 27,4 & & & & \\
\hline Perda & 125 & 36,9 & & & & \\
\hline \multicolumn{7}{|l|}{ Fuma } \\
\hline Não & 147 & 43,4 & & & & \\
\hline Sim & 33 & 9,7 & & & & \\
\hline Fumou & 159 & 46,9 & & & & \\
\hline \multicolumn{7}{|l|}{ Etilista } \\
\hline Não & 229 & 67,6 & & & & \\
\hline Sim & 32 & 9,4 & & & & \\
\hline Bebeu & 78 & 23,0 & & & & \\
\hline \multicolumn{7}{|l|}{ Atividade física } \\
\hline Não & 258 & 76,1 & & & & \\
\hline Sim & 81 & 23,9 & & & & \\
\hline
\end{tabular}

Fonte: Elaboração própria.

Quanto ao suporte de cuidado e social, 59 $(17,4 \%)$ idosos tinham cuidador, sendo $89,8 \%$ um familiar, principalmente filhos $(67,9 \%)$, seguido do cônjuge (18,9\%). A maior parte $(83,8 \%)$ dependia exclusivamente do SUS.
Na Tabela 3, observa-se que 27 entrevistados apresentavam ferida crônica, trazendo prevalência geral estimada de 8\% (IC95\%5,0-10,9), sendo que a de LP foi de 5\% (IC95\%2,9-7,7) e de UV 2,9\% (IC95\%1,2-4,7). 
Tabela 3 - Prevalência de feridas crônicas por tipo. Teresina, Piauí, Brasil - 2016. (n=339)

\begin{tabular}{|c|c|c|c|}
\hline Variáveis & $\mathbf{N}$ & $\%$ & $\begin{array}{c}\text { Intervalo de Confiança } \\
95 \%\end{array}$ \\
\hline \multicolumn{4}{|c|}{ Prevalência geral de ferida crônica } \\
\hline Não & 312 & 92,0 & $89,1-95,0$ \\
\hline Sim & 27 & 8,0 & $5,0-10,9$ \\
\hline \multicolumn{4}{|c|}{ Prevalência de lesão por pressão } \\
\hline Não & 322 & 95,0 & $92,3-97,1$ \\
\hline Sim & 17 & 5,0 & $2,9-7,7$ \\
\hline \multicolumn{4}{|c|}{ Prevalência de úlcera vasculogênica } \\
\hline Não & 329 & 97,1 & $95,3-98,8$ \\
\hline Sim & 10 & 2,9 & $1,2-4,7$ \\
\hline
\end{tabular}

Fonte: Elaboração própria.

Observa-se que 70,4\% dos idosos apresentavam mais de uma lesão, com média de 2,7 feridas por paciente $( \pm 1,4)$, totalizando 40 lesões. As localizações de maior frequência foram as regiões sacral $(32,5 \%)$ e terço distal da perna (15\%). Quanto às dimensões, 44\% tinham área superior a $24,0 \mathrm{~cm}^{2}$ e $29,7 \%$ área de 1,1 a 8,0 $\mathrm{cm}^{2}, 11,1 \%$ com grande quantidade de exsudato, $14,8 \%$ apresentando tecido do tipo esfacelo, com tempo médio de 30,6 meses $( \pm 41,1)$. Em relação ao escore de Push, 51,8\% estavam acima de 9. Das 40 lesões avaliadas, quanto ao estágio, a maioria $(42,9 \%)$ das LP era de estágio 3, seguida do estágio $4(32,1 \%)$ e não classificável (14,3\%); e as UV eram todas de espessura parcial.

Apresentaram associação estatística significativa com a ocorrência de ferida crônica, as variáveis escolaridade $(\mathrm{p}=0,03)$ e MEEM $(\mathrm{p}=0,000)$ (Tabela 4).

Tabela 4 - Associação da ocorrência de ferida crônica com variáveis sociodemográficas e clínicas. Teresina, Piauí, Brasil - 2016. (n=339)

(continua)

\begin{tabular}{|c|c|c|c|c|c|c|c|c|c|}
\hline \multirow{2}{*}{ Variáveis } & \multicolumn{4}{|c|}{ Presença de Ferida Crônica } & \multicolumn{2}{|c|}{ Total } & \multirow{2}{*}{ p-valor } & \multirow{2}{*}{$\begin{array}{l}\text { Odds } \\
\text { Ratio }\end{array}$} & \multirow{2}{*}{$\begin{array}{c}\text { Intervalo de } \\
\text { Confiança } \\
95 \%\end{array}$} \\
\hline & $\begin{array}{c}\text { Não } \\
\text { N }\end{array}$ & $\%$ & $\underset{\mathbf{N}}{\operatorname{Sim}}$ & $\%$ & $\mathbf{N}$ & $\%$ & & & \\
\hline Sexo & & & & & & & $0,525^{*}$ & 1,429 & $0,585-3,487$ \\
\hline Masculino & 104 & 30,7 & 7 & 2,1 & 111 & 32,7 & & & \\
\hline Feminino & 208 & 61,4 & 20 & 5,9 & 228 & 67,3 & & & \\
\hline Escolaridade & & & & & & & $\mathbf{0 , 0 3 *}$ & 0,289 & $0,120-0,696$ \\
\hline Sem & 125 & 44,3 & 24 & 8,5 & 149 & 52,8 & & & \\
\hline Com & 126 & 44,7 & 7 & 2,5 & 133 & 47,2 & & & \\
\hline Renda & & & & & & & $0,80 * *$ & 0,777 & $0,261-2,309$ \\
\hline Até 3 SM & 261 & 77 & 32 & 9,4 & 293 & 86,4 & & & \\
\hline$>3 \mathrm{SM}$ & 42 & 12,4 & 4 & 1,2 & 46 & 13,6 & & & \\
\hline $\begin{array}{l}\text { Toma } \\
\text { medicamento }\end{array}$ & & & & & & & $0,09^{* *}$ & 1,182 & $0,396-3,524$ \\
\hline Não & 39 & 11,5 & 4 & 1,2 & 43 & 12,7 & & & \\
\hline Sim & 264 & 77,9 & 32 & 9,4 & 296 & 87,3 & & & \\
\hline $\begin{array}{l}\text { Presença de } \\
\text { doença }\end{array}$ & & & & & & & $0,336^{* *}$ & 3,424 & $0,451-25,983$ \\
\hline Sim & 27 & 8,0 & 1 & 0,3 & 28 & 8,3 & & & \\
\hline Não & 276 & 81,4 & 35 & 10,3 & 311 & 91,7 & & & \\
\hline
\end{tabular}


Tabela 4 - Associação da ocorrência de ferida crônica com variáveis sociodemográficas e clínicas. Teresina, Piauí, Brasil - 2016. (n=339)

(conclusão)

\begin{tabular}{|c|c|c|c|c|c|c|c|c|c|}
\hline \multirow{2}{*}{ Variáveis } & \multicolumn{4}{|c|}{ Presença de Ferida Crônica } & \multicolumn{2}{|c|}{ Total } & \multirow{2}{*}{ p-valor } & \multirow{2}{*}{$\begin{array}{l}\text { Odds } \\
\text { Ratio }\end{array}$} & \multirow{2}{*}{$\begin{array}{c}\text { Intervalo de } \\
\text { Confiança } \\
95 \%\end{array}$} \\
\hline & $\begin{array}{c}\text { Não } \\
\mathbf{N}\end{array}$ & $\%$ & $\underset{\mathbf{N}}{\operatorname{Sim}}$ & $\%$ & $\mathbf{N}$ & $\%$ & & & \\
\hline \multicolumn{7}{|l|}{$\begin{array}{l}\text { Mini-Exame do } \\
\text { Estado Mental }\end{array}$} & $0,000^{*}$ & 7,189 & $3,436-15,043$ \\
\hline $\begin{array}{l}\text { Cognitivo } \\
\text { preservado }\end{array}$ & 266 & 78,5 & 18 & 5,3 & 284 & 83,8 & & & \\
\hline Déficit cognitivo & 37 & 10,9 & 18 & 5,3 & 55 & 16,2 & & & \\
\hline
\end{tabular}

Fonte: Elaboração própria.

* Qui-quadrado de Pearson; ** Teste exato de Fischer.

$\mathrm{SM}=$ Salário mínimo $(\mathrm{R} \$ 880,00)$.

Conforme Tabela 5, verifica-se que houve diferença estatística significativa na idade dos idosos quando comparados aqueles que tinham ferida com quem não tinha $(\mathrm{p}=0,000)$.

Tabela 5 - Comparação das médias da idade dos idosos entrevistados. Teresina, Piauí, Brasil - 2016. $(n=339)$

\begin{tabular}{l|c|c|c|c}
\hline Ferida crônica & n & Média da idade & Desvio Padrão & p-valor \\
\hline Não & 303 & 70,69 & 8,471 & $0,000^{*}$ \\
Sim & 36 & 74,33 & 12,066 & \\
\hline
\end{tabular}

Fonte: Elaboração própria.

*Teste t de Student.

\section{Discussão}

A maior concentração dos idosos do estudo encontrou-se na faixa etária de 60 a 70 anos. Isto evidencia recente processo de envelhecimento brasileiro, que se diferencia do que vem ocorrendo nos países desenvolvidos, cuja concentração é maior no grupo de 80 anos ou mais. As estimativas do último censo apontam que o grupo com idade igual ou superior a 80 anos será o que mais crescerá nos próximos anos, pois representava, em 2010, 1,53\% da população geral e têm-se expectativas que representarão $8,75 \%$ da população em $2060^{(12)}$.

A idade média de 71,1 anos confirma que a expectativa de vida da população brasileira tem aumentado ${ }^{(13)}$. Considerando que os idosos são os mais acometidos com feridas crônicas, pode-se afirmar que este fator torna-se relevante, uma vez que se verificou diferença na média de idade dos idosos, ao se comparar os que apresentavam alguma lesão com os que não tinha nenhuma. Além disso, pessoas que vivem mais, terão maior possibilidade de exposição aos fatores de risco.

A predominância do sexo feminino entre os idosos reflete o fenômeno denominado "feminização da velhice", que ocorre devido à expectativa de vida mais elevada entre mulheres. O contingente feminino de mais de 60 anos de idade passou de 2,2\%, em 1940, para 4,7\%, em 2000, e $6 \%$, em $2010^{(12)}$.

Em relação ao sexo, a diferença percentual entre feminino e masculino de indivíduos acometidos por feridas vem diminuindo ao longo dos anos, embora as mulheres ainda sejam as que apresentam maior número, devido a fatores como gravidez e hormônios ${ }^{(13-14)}$. Por muito tempo, a maioria dos estudos apontou maior 
ocorrência de úlceras entre o sexo feminino ${ }^{(14-15)}$. Entretanto, alguns estudos mostram maior ocorrência em pacientes do sexo masculino ${ }^{(16-17)}$. No presente estudo não se observou associação do sexo com a ocorrência de ferida crônica.

O maior índice de analfabetismo entre idosos é considerado reflexo das políticas educacionais brasileiras do passado. Nas décadas de 1930 a 1950, o ingresso nas instituições escolares era restrito a segmentos sociais específicos. Neste sentido, o baixo saldo da escolaridade média dessa população decorre desse acesso desigual $^{(18)}$. Assim, foi encontrada associação da escolaridade com a ocorrência de ferida crônica, o que evidencia tendência para o aumento da prevalência dessas lesões em idosos com baixo grau de instrução. Por essa razão, torna-se essencial considerar a avaliação do nível de escolaridade como fator importante para promoção de ações de atenção à saúde. Assim, os profissionais devem estar atentos à utilização de linguagem simples e acessível como meio facilitador da comunicação, no intuito de repassar informações sobre saúde a pessoas com feridas.

Parte dos idosos da pesquisa apresentava baixa renda familiar e realizava algum tipo de atividade ou trabalho. Na maioria dos casos, o idoso retornava ou continuava no mercado de trabalho para ajudar a complementar a renda familiar, pois, em inúmeras situações, o benefício da aposentadoria não era suficiente para cobrir os gastos pessoais. Também é uma forma de o idoso sentir-se útil, ocupar e se reconhecer como ser produtivo em uma sociedade capitalista ${ }^{(19)}$. Este fato aponta que os rendimentos são diminuídos entre os idosos, mesmo que os fatores socioeconômicos sejam importantes na vida diária e na qualidade de vida deste grupo populacional ${ }^{(14)}$. A renda não apresentou associação estatística com a ocorrência de ferida crônica neste estudo, mas também é um dado preocupante no contexto das feridas crônicas, pois a ausência de recursos financeiros para manutenção do tratamento pode acarretar o abandono da terapia pelo paciente.

Ter uma ou mais doenças crônicas caracterizou a amostra do estudo, destacando-se entre elas hipertensão arterial e diabetes, porém não apresentou associação estatística com a ocorrência de lesões crônicas. Entretanto, ao se agregar o MEEM com a ocorrência de ferida crônica na população estudada, constatou-se associação estatística significativa. Esse dado é importante, haja vista que a doença crônica provoca mudanças, especialmente na rotina e no planejamento de atividades, o que aumenta não só as responsabilidades como também as habilidades no cuidado da ferida ${ }^{(20)}$, o que é preocupante, quando se trata de um idoso com doenças incapacitantes que comprometem o estado cognitivo.

A maior parte deles fazia uso de uma ou mais medicações, prevalecendo também os antihipertensivos. Essa realidade também foi evidenciada em estudo epidemiológico de pacientes com feridas crônicas atendidos no ambulatório de um hospital universitário na região Sudeste do Brasil, em que a maioria referiu algum tipo de doença de base, principalmente diabetes ou hipertensão, doenças que são a causa do surgimento da ferida ou do retardo na cicatrização ${ }^{(13)}$.

Ainda nesse contexto, o uso contínuo de fármacos também deve ser avaliado. Os antihipertensivos, por exemplo, também predispõem ao desenvolvimento de LP e UV, pois reduzem o fluxo de perfusão sanguínea no tecido, favorecendo o aumento da sensibilidade do doente à pressão exercida. Desta forma, os enfermeiros devem direcionar a assistência, considerando os aspectos clínicos do paciente, os sinais e sintomas da patologia associada e os aspectos da ferida ${ }^{(13)}$.

Os resultados demostraram que os entrevistados não eram tabagistas ou etilistas. O fumo e o álcool também podem ser considerados fatores de risco, pois o primeiro produz efeitos no organismo que interferem no fluxo sanguíneo, provocando vasoconstrição, favorecendo a diminuição do aporte de oxigênio e nutrientes para as células, e o segundo pode ocasionar lesão de células neuronais, de sistema cardíaco, células hepáticas e pancreáticas ${ }^{(21)}$.

A maioria dos participantes não praticava atividade física. Como o sedentarismo mostra-se fortemente associado ao desenvolvimento de 
doenças crônicas, a prática de atividades físicas é apontada como importante estratégia para prevenção dessas doenças. Além disso, a atividade física pode prevenir a recorrência de feridas crônicas, como as úlceras venosas ${ }^{(22)}$.

No estudo, verificou-se que pequena parte dos idosos possuía cuidador, haja vista que a maioria era independente para realizar as atividades de vida diária e não tinha problemas de mobilidade. Os que tinham, eram, na maioria dos casos, um familiar. Além disso, a maioria dos entrevistados não morava sozinha e sim com cônjuge, filhos e/ou netos. A família é fonte de apoio informal aos idosos, o que se verifica em situações de corresidência ou não, quando os membros de uma família se ajudam, na busca do alcance do bem-estar coletivo(6).

A prevalência de feridas crônicas no estudo foi de 8\% (IC95\%5,0-10,9). Aumento da prevalência de feridas crônicas pode ser esperado, considerando as mudanças demográficas e o envelhecimento da população. A população idosa tem condições predisponentes para o desenvolvimento desses agravos, pois, além da prevalência das condições crônicas, que levam a um declínio da capacidade funcional, a própria fisiologia do envelhecimento causa diminuição da eficiência dos sistemas respiratório, circulatório, sensorial e nutricional, que gera comprometimento do fluxo sanguíneo, deficiente oxigenação, nutrição e hidratação dos tecidos e, consequentemente, contribui para aumentar esse risco ${ }^{(16,23)}$.

No entanto, os índices epidemiológicos variam consideravelmente, dependendo do diagnóstico, ano e país. Internacionalmente, existem grandes variações nos dados de prevalência e incidência de feridas crônicas. Na pesquisa realizada, a ferida mais prevalente foi a LP, com 5\% (IC95\% 2,9-7,7), seguida da UV com 2,9\% (IC95\% $1,2-4,7)$, dados que divergem de outro estudo, realizado em 2010 com idosos cadastrados na ESF de um município do interior de Goiás, em que a maior prevalência foi de feridas de etiologia vasculogênica, conhecidas como úlceras de perna, que constituem uma das patologias mais frequentes nos ambulatórios dos hospitais e em consultórios ${ }^{(15)}$.
Em estudo realizado em 2012, na Alemanha, as úlceras de perna foram os ferimentos mais frequentes, correspondendo a $64 \%$ de todas as feridas identificadas, seguidas por lesões por pressão (41\%) e úlceras diabéticas $(17 \%)^{(24)}$. No Brasil, em estudo epidemiológico realizado em ambulatório na região Sudeste em 2010, 51\% dos pacientes possuíam úlceras de etiologia venosa, $24 \%$ de etiologia diabética, 13\% de outras etiologias (lesões cirúrgicas), 6\% com lesão por pressão, 3\% com úlcera de etiologia neuropática, $2 \%$ com úlcera de etiologia arterial e 1\% sem informação da classificação ${ }^{(13)}$.

As inúmeras lesões de pele que afetam os idosos são apontadas com incidências distintas, relacionando o ambiente como fator somatório à integridade tissular prejudicada ${ }^{(15)}$. Em âmbito nacional, poucos estudos investigaram as feridas crônicas no domicílio. Em Ribeirão Preto, interior do estado de São Paulo, Brasil, uma investigação sobre as características sociodemográficas, clínicas e o risco para o desenvolvimento de LP, a prevalência encontrada foi de $17 \%$ naqueles com 60 anos ou mais ${ }^{(6)}$. Internacionalmente, um estudo verificou prevalência de LP em pessoas acima de 65 anos de 0,44\% (IC 95\%:0,41-0,47\%) $)^{(25)}$.

Em Teresina, Piauí, Brasil, a ESF enfrenta dificuldades, devido ao grande número de pacientes agregados à estrutura de famílias teresinenses, fora da programação das equipes, que não conseguem dar continuidade às ações programáticas de promoção da saúde e prevenção de doenças, desviando o foco para ações curativas. Frente a essas dificuldades, busca-se atender pessoas que se encontram com estado de saúde agravado e são admitidas na equipe, muitas vezes, em situação de imobilidade prolongada e com LP, o que gera indicadores de saúde irreais, por se tratar de pacientes provenientes de outros municípios, fato que aumenta o número de LP nas equipes da $\mathrm{ESF}^{(5)}$.

Associado a isso, há relacionamento linear entre o aumento da idade dos pacientes e o da ocorrência de LP, devido à prevalência de condições crônicas, que levam à diminuição da capacidade funcional nos idosos e à imobilidade ${ }^{(6)}$. 
Quanto à localização das lesões, a maioria dos idosos apresentava mais de uma e em diferentes localizações anatômicas. A região mais atingida foi a sacral, que se associa ao tipo mais prevalente de lesão, associada à posição em que geralmente se encontra o paciente acamado ou cadeirante. Este resultado corrobora os achados de outros estudos ${ }^{(5,23)}$.

Quanto aos tecidos encontrados nas feridas, o de granulação estava presente em 48,2\% dos casos. A característica do tecido no leito da ferida é importante indicador do estágio da cicatrização alcançado ou de complicações que possam estar presentes. O tecido de granulação no leito é um ponto positivo, por favorecer o reparo tecidual e, consequentemente, o fechamento da lesão ${ }^{(13)}$.

A média de tempo da existência das feridas crônicas foi de cerca de três anos, tempo prolongado previsto, por se tratar de lesões crônicas, ocorrendo a cicatrização em tempo mais prolongado que o habitual, além da possibilidade de recorrência dessas úlceras.

Muitas vezes, a demora na cicatrização de uma ferida pode estar associada a condições pré-existentes, como a hipertensão, o diabetes, estado nutricional inadequado, imunodeficiência ou infecção. Acrescenta-se, ainda, que o longo tempo transcorrido entre o surgimento de uma ferida e a sua cicatrização sofre influência da terapêutica adotada para o tratamento ${ }^{(14)}$. Tal situação pode ser evidenciada pelos escores de Push apresentados pela maioria dos casos, indicativos de condições ruins de cicatrização.

Diante do exposto, relembra-se o papel do enfermeiro na atenção básica, na qual desempenha trabalho importante ao tratar das feridas, pois é responsável por acompanhar a evolução da lesão, orientar e executar o curativo de forma eficiente e humanizada ${ }^{(26)}$. Além disso, destaca-se o seu papel na responsabilidade de manter a observação intensiva com relação aos fatores que condicionam o surgimento e evolução da ferida, principalmente se tratando da pessoa idosa.

\section{Conclusão}

Os resultados deste estudo mostraram que os idosos apresentam-se cada vez mais vulneráveis para o desenvolvimento de feridas crônicas, pois foi constatada diferença na média da idade, ao se comparar idosos portadores de LP e UV com idosos que não apresentavam essas lesões, o que pode ser explicado pela presença de morbidades múltiplas, condições clínicas crônicas que aumentam o risco para ocorrência de feridas crônicas. Tais condições, associadas às características sociodemográficas e clínicas, como baixa escolaridade e comprometimento cognitivo, favorecem tanto o aparecimento dessas feridas como pode interferir no processo de cicatrização da lesão.

É importante o levantamento da prevalência desses agravos na população idosa, bem como o conhecimento do perfil desses idosos, para o planejamento do cuidado a ser prestado na atenção básica. Assim, a presente pesquisa contribuirá para melhoria da atuação dos profissionais de saúde da atenção básica, de forma especial o enfermeiro, que lida corriqueiramente com a prestação do cuidado ao idoso e tem a responsabilidade de buscar atualizações e aplicar na prática medidas preventivas e protetoras para ocorrência de feridas crônicas.

\section{Colaborações:}

1. concepção, projeto, análise e interpretação dos dados: Chrystiany Plácido de Brito Vieira, Alessandra Silveira Furtado, Priscilla Carvalho Dias de Almeida e Maria Helena Barros Araújo Luz;

2. redação do artigo e revisão crítica relevante do conteúdo intelectual: Chrystiany Plácido de Brito Vieira, Alessandra Silveira Furtado, Priscilla Carvalho Dias de Almeida e Antônio Francisco Machado Pereira;

3. aprovação final da versão a ser publicada: Chrystiany Plácido de Brito Vieira, Alessandra Silveira Furtado e Priscilla Carvalho Dias de Almeida. 


\section{Referências}

1. Choi EP, Chin WY, Wan EY, Lam CL. Evaluation of the internal and external responsiveness of the Pressure Ulcer Scale for Healing (PUSH) tool for assessing acute and chronic wounds. J Adv Nurs. 2016 May;72(5):1134-43.

2. Gould L, Abadir P, Brem H, Carter M, Conner-Kerr $\mathrm{T}$, Davidson J, et al. Chronic wound repair and healing in older adults: current status and future research. J Am Geriatr Soc. 2015 Mar;63(3):427-38.

3. McDaniel JC, Kemmner KG, Rusnak S. Nutritional profile of older adults with chronic venous leg ulcers: a pilot study. Geriatr Nurs. 2015 Sept-Oct;36(5):381-6.

4. Gomes T, Cade NV, Rohr RV, Fejoli MM. Caracterização das lesões crônicas e os fatores associados em moradores de um território de saúde em Vitória, Espírito Santo. Rev Bras Pesq Saúde. 2011;13(1):52-7.

5. Bezerra SMG, Luz MHBA, Andrade EMLR, Araújo TME, Teles JBM, Caliri MHL. Prevalência, fatores associados e classificação de úlcera por pressão em pacientes com imobilidade prolongada assistidos na Estratégia Saúde da Família. Rev Estima. 2014;12(3):41-9.

6. Chayamiti EMPC, Caliri MHL. Pressure ulcer in patients under home care. Acta Paul Enferm. 2010;23(1):29-34.

7. Bertolucci PHF, Brucki SMD, Campacci SR, Juliano Y. O mini-exame do estado mental em uma população geral. Impacto da escolaridade. Arq Neuropsiquiatr. 1994;52(1):1-7.

8. Lino VTS, Pereira SRM, Camacho LAB, Ribeiro Filho ST, Buksman S. Adaptação transcultural da Escala de Independência em Atividades de Vida Diária (Escala de Katz). Cad saúde pública. 2008;24(1):103-12.

9. National Pressure Ulcer Advisory Panel. Technical note: change in terminology from pressure ulcer to pressure injury and updates the stages of pressure injury [Internet]. New York; 2016. [cited 2016 Feb 20]. Available from: http://www.npuap. org/resources/educational-and-clinical-resources/ npuap-pressure-ulcer-stagescateg

10. Côrtes SMS. O tratamento de ferida: um artigo de revisão. REVISA. 2013;2(1):55-64.

11. Santos VLCG, Azevedo MAJ, Silva TS, Carvalho VM, Carvalho VF. Adaptação transcultural do
Pressure Ulcer Scale for Healing (PUSH), para a língua portuguesa. Rev Latino-am Enfermagem. 2005 maio-jun;13(3):305-13.

12. Instituto Brasileiro de Geografia e Estatística. Sinopse dos resultados do censo 2010 [internet]. Rio de Janeiro; 2010 [cited 2015 Mar 3]. Available from: htpp://www.censo2010.ibge.gov.br/sinopse/ webservice

13. Oliveira BGRB, Castro JBA, Granjeiro JM. Panorama epidemiológico e clínico de pacientes com feridas crônicas tratados em ambulatório. Rev Enferm UERJ. 2013;21(5):612-7.

14. Evangelista DG, Magalhães ERM, Moretão DIC, Stival MM, Lima LR. Impacto das feridas crônicas na qualidade de vida de usuários da estratégia de saúde da família. Rev Enferm Cent-Oeste Min. 2012;2(2):254-63.

15. Cavalcante AMRZ, Moreira A, Azevedo KB, Lima LR, Coimbra WKAM. Diagnóstico de enfermagem: integridade tissular prejudicada identificado em idosos na Estratégia de Saúde da Família. Rev eletrônica enferm [Internet]. 2010;12(4):727-35. Available from: http://dx.doi.org/10.5216/ree. v12i4.8425

16. Sant'Ana SMSC, Bachion MM, Santos QR, Nunes CAB, Malaquias SG, Oliveira BGRB. Úlceras venosas: caracterização clínica e tratamento em usuários atendidos em rede ambulatorial. Rev bras enferm. 2012 ago;65(4):637-644.

17. Haji Zaine N, Burns J, Vicaretti M, Fletcher JP, Begg L, Hitos K. Characteristics of diabetic foot ulcers in Western Sydney, Australia. J Foot Ankle Res. 2014;7(1):39.

18. Soares MRP, Istoe RSC. Alfabetização e inclusão de pessoas idosas: uma proposta interdisciplinar mediada pelas tecnologias da informação e da comunicação. Rev Cient Interdisc. 2015;2(3):165-75.

19. Muniz TS, Barros A. Otrabalhadoridoso no mercado de trabalho do capitalismo contemporâneo. Rev Ciênc Humanas Soc. 2014;2(1):103-16.

20. Waidman MAP, Rocha SC, Correa JL, Brischiliari A, Marcon SS. O cotidiano do indivíduo com ferida crônica e sua saúde mental. Texto Contexto Enferm. 2011 Out-Dez;20(4):691-9.

21. Araújo RO, Silva DC, Souto RQ, Pergola-Marconato AM, Costa IKF, Torres GV. Impacto de úlceras venosas na qualidade de vida de indivíduos atendidos na atenção primária. Aquichán. 2016 Jan;16(1):56-66. 
22. Finlayson K, Wu ML, Edwards HE. Identifying risk factors and protective factors for venous leg ulcer recurrence using a theoretical approach: A longitudinal study. Int J Nurs Stud. 2015 Jun; 52(6):1042-51.

23. Pessoa EFR, Rocha JGSC, Bezerra SMG. Prevalência de úlcera por pressão em pacientes acamados, cadastrados na Estratégia de Saúde da Família: um estudo de enfermagem. Rev Interdisciplinar NOVAFAPI. 2011;4(1):14-8.

24. Heyer K, Herberger K, Protz K, Glaeske G, Augustin M. Epidemiology of chronic wounds in Germany: analysis of statutory health insurance data. Wound Repair Regen. 2016 Mar;24(2):434-42.
25. Pancorbo-Hidalgo PL, García-Fernández FP, Torra i Bou JE, Verdú Soriano J, Soldevilla-Agreda JJ. Epidemiología de las úlceras por presión en España en 2013: 4..$^{\circ}$ Estudio Nacional de Prevalencia. Gerokomos. 2014;25(4):162-70.

26. Lara MO, Pereira Júnior AC, Pinto JSF, Vieira NF, Wichr P. Significado da ferida para portadores de úlceras crônicas. Cogitare Enferm. 2011 jul/ set;16(3):471-7.

Recebido: 4 de agosto de 2016

Aprovado: 14 de agosto de 2017

Publicado: 4 de outubro de 2017 